\title{
Dietary diversity, vitamin $D$ intake and childhood stunting: a case-control study in Bantul, Indonesia
}

\author{
Eka Nurhayati ${ }^{1^{*}}$, Bunga Astria Paramashanti ${ }^{2}$, Dewi Astiti ${ }^{2}$ \& Arif Sabta Aji ${ }^{2}$ \\ ${ }^{1}$ Department of Midwifery, Universitas Alma Ata, Yogyakarta, Indonesia; ${ }^{2}$ Department \\ of Nutrition, Universitas Alma Ata, Yogyakarta, Indonesia
}

\begin{abstract}
Introduction: Stunting is known to be a major public health problem among Indonesian children. We aimed to examine the association between dietary diversity and vitamin D intake with stunting in children aged 6-23 months. Methods: This case-control study was conducted in Bantul District, Yogyakarta Special Region, Indonesia. A total of 79 subjects aged 6-23 months were selected for each case and control group based on their stunting status. We assessed potential explanatory variables at the child, parental, household, and community levels. Results: Factors which were significantly associated with stunting included young children aged 1823 months (adjusted $O R=3.84$; 95\% CI: 1.17-12.26), birth length $\geq 48 \mathrm{~cm}$ (adjusted $O R=0.36$; 95\% CI: 0.16-0.83), inadequate intake of vitamin D (adjusted $O R=5.18$; 95\% CI: 1.03-26.02), and diversified diet (adjusted OR $=0.17$; 95\% CI: 0.03-0.92). Other variables such as household economic status, living residency, history of exclusive breastfeeding, and infectious diseases, as well as intakes of energy and protein were not significantly related to stunting. Conclusion: Minimum dietary diversity, vitamin D intake from complementary foods, and birth length were associated with stunting status among children. Therefore, it is crucial to focus on stunting prevention programmes in the first two years of life, or even since the preconception period.
\end{abstract}

Keywords: Stunting, dietary diversity, vitamin D, determinants, Indonesia

\section{INTRODUCTION}

Stunting is a public health problem in Indonesian children. According to the Indonesian National Basic Health Survey in 2018, the prevalences of stunting and severe stunting in children under two years old were $17.1 \%$ and $12.8 \%$, respectively (NIHRD, 2019), which meant that one in three Indonesian children under 2-year-old experienced chronic undernutrition. Stunting has been associated with increased adverse effects including failure of reaching growth potential, decreased neurocognitive functions, and greater risk of obesity and non-communicable diseases in later life (de Onis \& Branca, 2016). In addition, it is responsible for $14.5 \%$ of deaths and $12.6 \%$ of disability-adjusted life-years (DALY) in children under the age of 5 years (Black et al., 2008).

Stunting is an interlinked process of growth which may begin in utero, then continue to neonatal, infant and childhood, pubertal, and adulthood, where each stage is influenced by different mechanisms (Prendergast \& Humphrey, 2014). Nonetheless, it is important to

\footnotetext{
*Corresponding author: Eka Nurhayati

Department of Midwifery, Universitas Alma Ata Yogyakarta, Indonesia

Tel: (+62)(274)434 2288; Fax: (+62)(274)4342269; E-mail: ekanhayati@almaata.ac.id doi: https://doi.org/10.31246/mjn-2020-0021
} 
realise that for a nutritional challenge such as stunting, its causes are deeply embedded in situational structures. A previous review on stunting determinants among Indonesian children highlighted that birth outcomes, infant and young child feeding practices, hygiene and sanitation, and sociodemographic factors may influence childhood stunting (Beal et al., 2018).

A large-scale study in Indonesia found that the odds of stunting was greater in children who were living in a household with three or more toddlers, had five to seven household members, mothers who attended $<4$ times of antenatal care during pregnancy, boys, and children who were born $<2500 \mathrm{~g}$ in weight (Titaley et al., 2019). However, another similar study concluded that feeding factors such as exclusive breastfeeding were not related to childhood stunting (Paramashanti, Hadi \& Gunawan, 2015). Specifically, an earlier study conducted in Yogyakarta Special Region reported that one of key determinants of childhood stunting included poor dietary diversity (Paramashanti, Paratmanitya \& Marsiswati, 2017).

Vitamin D intake may have a potential effect on stunting prevention and treatment (Yu et al., 2017). However, none of the previous studies in Indonesia showed this significant association (Ramadhani, Bahar \& Dachlan, 2019; Chairunnisa, Kusumastuti \& Panunggal, 2018). Due to the inconsistent results and limited variables used, as well as the cross-sectional designs used by most of these studies, we could not draw any conclusion.

Bantul is one of the five districts/ municipalities in Yogyakarta Special Region recruited by the Indonesian government as an intervention area to resolve stunting, even though the prevalence of stunting in this area is not so high. In the national programme of tackling stunting, our local government initiated a nutrition-improved village model in several regions in Yogyakarta Special Region. The public health office of Yogyakarta Special Region made an additional nutrition programme for pregnant women and children by giving fortified biscuits especially in areas with high malnutrition cases. The prevalence of stunting among children under 5 years old for Bantul District in 2017 was $10.41 \%$, the lowest prevalence amongst other districts in Yogyakarta Province (MOH Indonesia, 2017). A previous study showed that the odds of stunting increased significantly in children aged 12-24 months (Titaley et al., 2019), but there are no public data available yet about the prevalence of stunting for children under 2 years old for Bantul District.

Reducing stunting from a not-sohigh prevalence sounds challenging, therefore it is necessary to identify the proper risk factors in order to achieve this target. The intervention to reduce stunting will address some of the risk factors mentioned above, but also some other important risk factors that have not been investigated yet. Previous studies that have investigated the risk factors of stunting among Indonesian children had limitations such as unavailability of children's nutrient intake data, infectious diseases being limited only to diarrhoea in the past two weeks before data collection, and child's length at birth was not analysed (Beal et al., 2018; NIHRD, 2013). Therefore, this study aimed to examine whether dietary diversity and vitamin $\mathrm{D}$ intake were associated with stunting in children under the age of 2 years in Bantul District, Yogyakarta Special Region, Indonesia.

\section{MATERIALS AND METHODS}

A case-control study was conducted in Bantul District, Yogyakarta Special Region, Indonesia. The Yogyakarta 
Special Region is located $565 \mathrm{~km}$ from Jakarta, the capital city of Indonesia. The region consists of five municipalities / districts: Bantul, Sleman, Kulonprogo, Gunungkidul, and Yogyakarta City. Specifically, the Bantul District was selected as the study location as it mirrored urban and rural areas. This study was done between July and September 2019.

The population in this study were young children aged 6-23 months residing in Bantul District, with samples recruited under the areas of Sewon, Pandak, and Bambanglipuro subdistricts. We defined case as a child whose height-for-age $\mathrm{Z}$-score was $<-2$ $\mathrm{SD}$, whereas control was a child with a height-for-age $Z$-score $\geq-2 \mathrm{SD}$, based on the World Health Organization (WHO) growth chart (WHO, 2006). For both cases and controls, we excluded children who did not own a maternal and child health book, were not de jure residents and diagnosed with congenital diseases that limited them for height measurement.

Sample size was calculated using OpenEpi version 3 with proportions of non-exclusive breastfeeding based on a previous study (Paramashanti et al., 2015). We set 95\% level of confidence $(\mathrm{CI}), 80 \%$ power, and $1: 1$ ratio between case and control groups within the Kelsey formula. Thus, we obtained 80 subjects for each case and control group. Samples were selected by using multistage cluster sampling, where we divided our samples based on sub-districts as clusters. Firstly, three of 17 sub-districts were chosen randomly, namely Sewon, Pandak, and Bambanglipuro sub-districts. Secondly, two villages were selected randomly by lottery in every sub-district to represent each the rural and urban areas. Lastly, we obtained the village-level nutritional status data listed in the February 2019 report from each village's primary health centre.

To minimise potential bias in this study, we reassessed the lengths of children to determine stunting status. In this study, 37 Posyandu (integrated health posts at the village level) were identified to be included. As Posyandu was held routinely to monitor children's body weight, to provide supplementary foods for wasted children, and to deliver counselling for mothers, thus it was feasible to interview mothers and to measure their child's height during data collection. Then, we differed children based on their stunting status. A computerised simple random sampling was used to select cases in each Posyandu, whereas controls were chosen within the same Posyandu.

Screenings from public health centres of both case and control groups showed that we obtained 122 stunted and 718 normal children. We randomly selected 80 subjects for each group. Unfortunately, one subject from the case group was excluded due to incorrect measurement result, thus resulting in only 79 children for each group (a total of 158 children) being analysed (Figure 1).

The primary outcome of this study was childhood stunting. A child was considered as stunted if the heightfor-age $\mathrm{Z}$-score was <-2 SD below the growth reference curve (WHO, 2006). At the time of the interview, the length of the child was measured by using an infantometer. Meanwhile, our predictors were dietary diversity and intake of vitamin $\mathrm{D}$ among the children. Dietary diversity is defined as the consumption of at least four food groups of a total of seven food groups: 1) grains, roots and tubers; 2) legumes and nuts; 3) dairy products; 4) flesh foods; 5) eggs; 6) vitamin-A rich fruits and vegetables; and 7 ) other fruits and vegetables (WHO, 2008). Dietary diversity was estimated by a semi-quantitative food frequency 


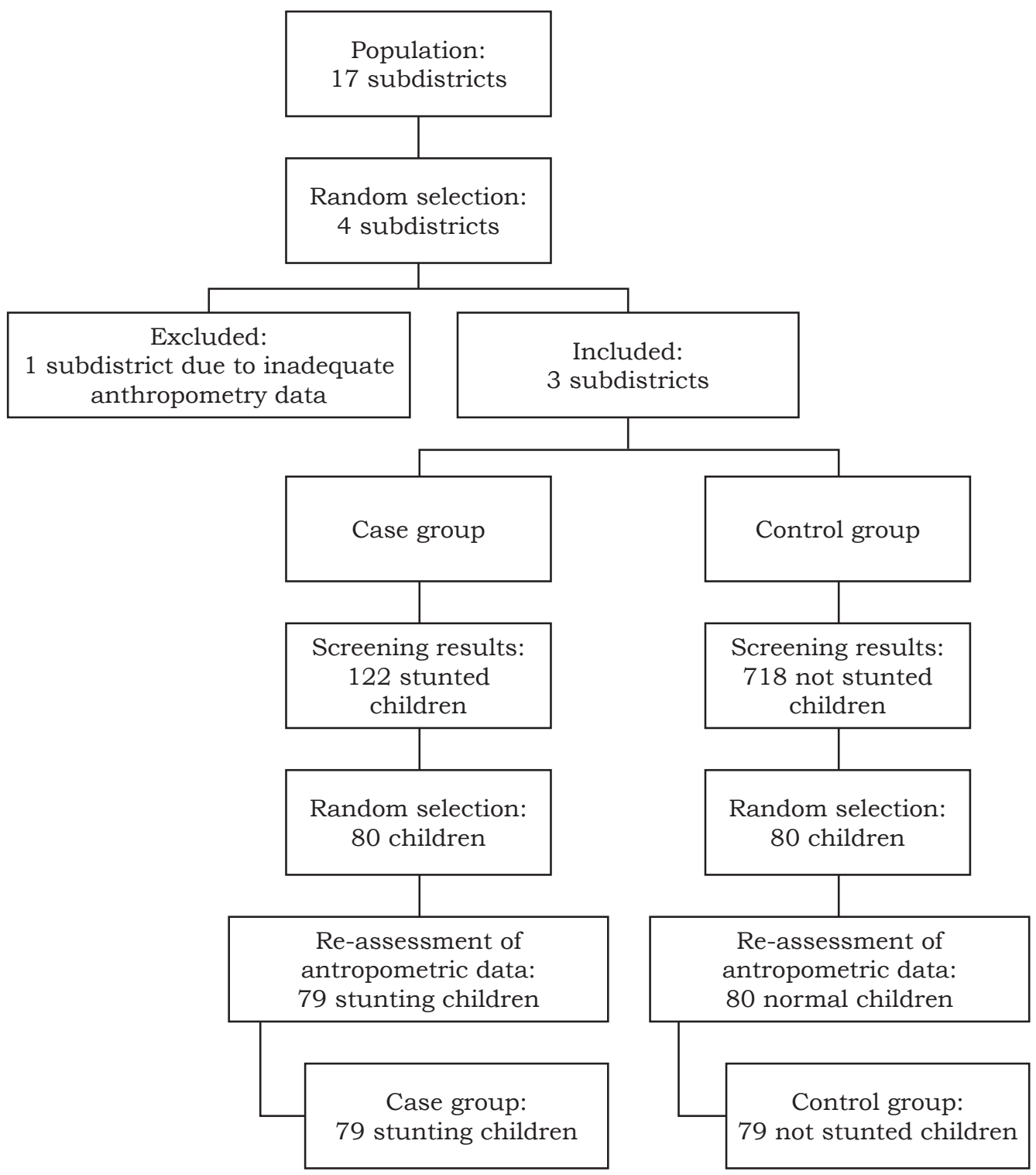

Figure 1. Diagram of case-control study subjects

questionnaire (semi-FFQ) with a threemonth timeframe. A minimum of ten grams per day was considered as consumption of each food item. Using the same semi-FFQ, intake of vitamin $\mathrm{D}$ was assessed using the Nutrisurvey Indonesia software and compared to the Recommended Dietary Allowance (RDA) of Indonesian population ( $\mathrm{MOH}$
Indonesia, 2013). We set a cut-off of $80 \%$ to categorise vitamin $\mathrm{D}$ intake.

Other variables collected in this study included child, parental, household, and community factors. Child factors were sex, age, feeding practices, infections, and history of birth outcomes. Infectious diseases were the occurrence of fever, cough, flu, and diarrhoea for the last 
one month. Feeding practices included history of exclusive breastfeeding, timing of introduction to complementary foods, and intakes of energy, protein, calcium, iron, phosphorus, and animal source foods. Animal source foods intake was the consumption of at least three of a total of six food groups: 1) breast milk and any other milk from animals; 2) other dairy products; 3) flesh foods; 4) organs; 5) fish or shellfish; and 6) eggs (Sebayang et al., 2019). Birth outcomes which included birth length, birth weight, and gestational age at birth were collected by enumerator's observation on the maternal and child health book. Parental factors were education levels of mothers and fathers. Educational attainment was considered as high if mothers or fathers completed at least senior high school education. Household and community factors included the number of children under 5 years, household economic status, and ruralurban living residency. Household economic status was calculated based on the household expenditure, then divided into three tertiles, which were poor, middle, and rich.

We described child, parental, household and community factors by using descriptive statistics. To analyse the relationship between each variable and stunting, we used the univariate logistic regression. All variables with a $p$-value $<0.25$ were included in the multiple logistic regression that controlled for the undesirable effects of potential confounding factors. A significance level was set at $5 \%$ to determine significant predictors. All analyses were performed by using STATA 14.2 (Stata Corporation, College Station, TX).

\section{Ethical statements}

This study was conducted according to the guidelines laid out in the Declaration of Helsinki and all procedures involving research study participants were approved by Alma Ata University Research Ethics Committee (No: KE/ AA/VII/986/EC/2019). All mothers in this study gave their written informed consent and subject anonymity data form prior to data collection.

\section{RESULTS}

\section{Population characteristics}

A total of 158 children ( 79 cases and 79 controls) were included in this study. More than half of the cases were males $(54.4 \%)$, whereas controls were females (53.2\%). Majority of children, 71 (89.9\%) cases and $62(74.5 \%)$ controls, were aged between 12-23 months. Mothers of 70 $(88.6 \%)$ cases and controls, and fathers of $61(77.2 \%)$ cases and $65(82.3 \%)$ controls completed senior high school education. Majority of cases (84.8\%) and controls $(88.5 \%)$ had one child under the age of 5 years within their household (Table 1).

\section{The differentiation of covariate variables in stunted and non-stunted children}

More than half of the stunted children had a birth length $<48 \mathrm{~cm}$, whereas $67.1 \%$ non-stunted children had a birth length $\geq 48 \mathrm{~cm}$. Approximately $90 \%$ of both cases and controls were born with normal birth weights ( $\geq 2500 \mathrm{~g}$ ) and term gestational age ( $\geq 37$ weeks). Seventy-six $(96.2 \%)$ cases and controls ever received breastmilk. Exclusive breastfeeding proportion was $64.6 \%$ among cases and $70.9 \%$ among controls. Majority of young children had adequate intakes of energy $(70.9 \%$ and $79.8 \%$, respectively in cases and in controls) and protein $(81.0 \%$ and $84.8 \%$, respectively in cases and in controls) from complementary foods (Table 2).

\section{Factors associated with stunting among young children}

Univariate logistic regression between 
Table 1. Socio-demographic characteristics of young children in Bantul District

\begin{tabular}{|c|c|c|c|}
\hline Characteristics & $\begin{array}{c}\text { Stunted }(n=79) \\
n(\%)\end{array}$ & $\begin{array}{c}\text { Not stunted }(n=79) \\
n(\%)\end{array}$ & $p$ \\
\hline \multicolumn{4}{|l|}{ Child factors } \\
\hline Sex & & & 0.340 \\
\hline Male & $43(54.4)$ & $37(46.8)$ & \\
\hline Female & $36(45.6)$ & $42(53.2)$ & \\
\hline Child's age & & & 0.000 \\
\hline 6-11 months & $8(10.1)$ & $17(21.6)$ & \\
\hline 12-17 months & $15(19.0)$ & $31(39.2)$ & \\
\hline $18-23$ months & $56(70.9)$ & $31(39.2)$ & \\
\hline \multicolumn{4}{|l|}{ Parental factors } \\
\hline Mother's education level & & & 1.000 \\
\hline Not completed elementary school & $0(0.0)$ & $1(1.3)$ & \\
\hline Completed elementary school & $5(6.3)$ & $2(2.5)$ & \\
\hline Completed junior high school & $4(5.1)$ & $6(7.6)$ & \\
\hline Completed senior high school & $50(63.3)$ & $46(58.2)$ & \\
\hline Completed tertiary education & $20(25.3)$ & $24(30.4)$ & \\
\hline Father's education level & & & 0.428 \\
\hline Not completed elementary school & $1(1.3)$ & $0(0.0)$ & \\
\hline Completed elementary school & $2(2.5)$ & $2(2.5)$ & \\
\hline Completed junior high school & $15(19.0)$ & $12(15.2)$ & \\
\hline Completed senior high school & $51(64.5)$ & $52(65.8)$ & \\
\hline Completed tertiary education & $10(12.7)$ & $13(16.5)$ & \\
\hline \multicolumn{4}{|l|}{ Household and community factors } \\
\hline $\begin{array}{l}\text { Number of children under } 5 \text { within } \\
\text { household }\end{array}$ & & & 0.502 \\
\hline 1 & $67(84.8)$ & $69(88.5)$ & \\
\hline$\geq 2$ & $12(15.2)$ & $9(11.5)$ & \\
\hline Household economic status & & & 0.505 \\
\hline Poor & $31(40.2)$ & $21(28.0)$ & \\
\hline Middle & $24(31.2)$ & $26(34.7)$ & \\
\hline Rich & $22(28.6)$ & $28(37.3)$ & \\
\hline Living residency & & & 0.203 \\
\hline Rural & $44(55.7)$ & $36(45.6)$ & \\
\hline Urban & $35(44.3)$ & $43(54.4)$ & \\
\hline
\end{tabular}

potential associated determinants and status of stunting in children are shown in Table 3. This study showed that children 18-23 months old (COR: 3.84, $p=0.005$ ) and with birth length $\geq 48 \mathrm{~cm}$ (COR:0.47, $p=0.022$ ) were associated with stunting. However, after using adjusted odds ratio, children 18-23 months old (AOR: 3.80, 
Table 2. Distribution of covariates across stunted and non-stunted young children in Bantul District

\begin{tabular}{|c|c|c|c|}
\hline Variables & $N$ & $\begin{array}{c}\text { Stunted } \\
n(\%)\end{array}$ & $\begin{array}{c}\text { Not stunted } \\
n(\%)\end{array}$ \\
\hline \multicolumn{4}{|l|}{ Birth outcomes } \\
\hline Birth length & 76 & & \\
\hline$<48 \mathrm{~cm}$ & & 39 (51.3) & $25(32.9)$ \\
\hline$\geq 48 \mathrm{~cm}$ & & $37(48.7)$ & $51(67.1)$ \\
\hline Birth weight & 76 & & \\
\hline$<2500 \mathrm{~g}$ & & $7(9.2)$ & $5(6.3)$ \\
\hline$\geq 2500 \mathrm{~g}$ & & $69(90.8)$ & $74(93.7)$ \\
\hline Gestational age & 76 & & \\
\hline$<37$ weeks & & $6(10.0)$ & $6(7.9)$ \\
\hline$\geq 37$ weeks & & $70(90.0)$ & $70(92.1)$ \\
\hline \multicolumn{4}{|l|}{ Feeding practices } \\
\hline Prelacteal & 79 & & \\
\hline Yes & & $6(7.6)$ & 7 (8.9) \\
\hline No & & $73(92.4)$ & $72(91.1)$ \\
\hline Ever breastfed & 79 & & \\
\hline Yes & & $76(96.2)$ & $76(96.2)$ \\
\hline No & & $3(3.8)$ & $3(3.8)$ \\
\hline Exclusive breastfeeding & 79 & & \\
\hline Yes & & $51(64.6)$ & $56(70.9)$ \\
\hline No & & $28(35.4)$ & $23(29.1)$ \\
\hline Introduction of complementary food & 79 & & \\
\hline$<6$ months & & $20(25.3)$ & $16(20.2)$ \\
\hline$\geq 6$ months & & $59(74.7)$ & $63(79.8)$ \\
\hline Energy intake from complementary food & 79 & & \\
\hline$<80 \%$ RDA & & $23(29.1)$ & $16(20.2)$ \\
\hline$\geq 80 \% \mathrm{RDA}$ & & $56(70.9)$ & $63(79.8)$ \\
\hline Protein intake from complementary food & 79 & & \\
\hline$<80 \% \mathrm{RDA}$ & & $15(19.0)$ & $12(15.2)$ \\
\hline$\geq 80 \% \mathrm{RDA}$ & & $64(81.0)$ & $67(84.8)$ \\
\hline Calcium intake from complementary food & 79 & & \\
\hline$<80 \% \mathrm{RDA}$ & & $50(63.3)$ & $45(57.0)$ \\
\hline$\geq 80 \% \mathrm{RDA}$ & & $29(36.7)$ & $34(43.0)$ \\
\hline Iron intake from complementary food & 79 & & \\
\hline$<80 \%$ RDA & & $42(53.2)$ & $45(57.0)$ \\
\hline$\geq 80 \% \mathrm{RDA}$ & & 37 (46.8) & $34(43.0)$ \\
\hline
\end{tabular}


Table 2. Distribution of covariates across stunted and non-stunted young children in Bantul District [Cont'd]

\begin{tabular}{|c|c|c|c|}
\hline Variables & $N$ & $\begin{array}{c}\text { Stunted } \\
n(\%)\end{array}$ & $\begin{array}{c}\text { Not stunted } \\
n(\%)\end{array}$ \\
\hline Phosphorus intake from complementary food & 79 & & \\
\hline$<80 \% \mathrm{RDA}$ & & $25(31.6)$ & $23(29.1)$ \\
\hline$\geq 80 \% \mathrm{RDA}$ & & $54(68.4)$ & $56(70.9)$ \\
\hline Vitamin D intake from complementary food & 79 & & \\
\hline$<80 \% \mathrm{RDA}$ & & $75(94.9)$ & $68(86.1)$ \\
\hline$\geq 80 \% \mathrm{RDA}$ & & $4(5.1)$ & $11(13.9)$ \\
\hline Dietary diversity & 79 & & \\
\hline$<4$ food groups & & 7 (8.9) & $4(5.1)$ \\
\hline$\geq 4$ food groups & & $72(91.1)$ & 75 (94.9) \\
\hline Animal source food & 79 & & \\
\hline$<3$ food groups & & $47(59.5)$ & 39 (49.4) \\
\hline$\geq 3$ food groups & & $32(40.5)$ & $40(50.6)$ \\
\hline \multicolumn{4}{|l|}{ Infectious diseases } \\
\hline Fever & 79 & & \\
\hline Yes & & $15(19.0)$ & $10(12.7)$ \\
\hline No & & 64 (81.0) & 69 (87.3) \\
\hline Cough & 79 & & \\
\hline Yes & & $10(12.7)$ & 9 (11.4) \\
\hline No & & 69 (87.3) & 70 (88.6) \\
\hline Flu & 79 & & \\
\hline Yes & & $12(15.2)$ & $10(12.7)$ \\
\hline No & & $67(84.8)$ & $69(87.3)$ \\
\hline Diarrhoea & 79 & & \\
\hline Yes & & $4(5.1)$ & $5(6.3)$ \\
\hline No & & 75 (94.9) & 74 (93.7) \\
\hline
\end{tabular}

RDA: recommended dietary allowance

$p=0.026)$, vitamin D intake $<80 \%$ of RDA from complementary foods (AOR: 5.18, $p=0.046)$, dietary diversity status $\geq 4$ food groups (AOR: 0.17, $p=0.040$ ), and birth length $\geq 48 \mathrm{~cm}(A O R: 0.36, p=0.016)$ were associated with stunting. Older children had a four-fold increased risk to be stunted than 6-11 months old children. Longer birth lengths had a protective effect against stunting in children, with a two-fold increased chance of being non-stunted compared to children who had shorter birth lengths. Similar result was found in the association between birth length and stunting in children even after adjusting for confounding variables. Children who had lower vitamin $\mathrm{D}$ intakes from complementary feeding had about five times higher risks of developing stunting than children who had $\geq 80 \%$ RDA of vitamin D intake. Moreover, a protective effect was found 
Table 3. Bivariate and multivariate results of factors associated with stunting

\begin{tabular}{|c|c|c|c|c|}
\hline Variables & COR & $p$ & $A O R$ & $p$ \\
\hline \multicolumn{5}{|l|}{ Child factors } \\
\hline \multicolumn{5}{|l|}{ Sex } \\
\hline \multicolumn{5}{|l|}{ Male (ref) } \\
\hline Female & $0.74(0.40-1.38)$ & 0.340 & & \\
\hline \multicolumn{5}{|l|}{ Child's age } \\
\hline \multicolumn{5}{|c|}{ 6-11 months (ref) } \\
\hline $12-17$ months & $1.03(0.36-2.92)$ & 0.958 & $0.80(0.22-2.89)$ & 0.733 \\
\hline $18-23$ months & $3.84(1.49-9.91)$ & $0.005^{*}$ & $3.80(1.17-12.26)$ & $0.026^{*}$ \\
\hline \multicolumn{5}{|c|}{ Parental and household factors } \\
\hline \multicolumn{5}{|c|}{ Mother's education level } \\
\hline \multicolumn{5}{|l|}{ Low (ref) } \\
\hline High & $1.00(0.38-2.67)$ & 1.000 & & \\
\hline \multicolumn{5}{|c|}{ Father's education level } \\
\hline \multicolumn{5}{|l|}{ Low (ref) } \\
\hline High & $0.73(0.33-1.59)$ & 0.429 & & \\
\hline \multicolumn{5}{|c|}{ Household and community factors } \\
\hline \multicolumn{5}{|c|}{$\begin{array}{l}\text { Number of children under } 5 \\
\text { within household }\end{array}$} \\
\hline \multicolumn{5}{|l|}{1 (ref) } \\
\hline$\geq 2$ & $1.37(0.54-3.47)$ & 0.503 & & \\
\hline \multicolumn{5}{|c|}{ Household economic status } \\
\hline \multicolumn{5}{|l|}{ Poor (ref) } \\
\hline Middle & $0.63(0.29-1.37)$ & 0.240 & $0.66(0.25-1.80)$ & 0.419 \\
\hline Rich & $0.53(0.24-1.17)$ & 0.116 & $0.69(0.26-1.85)$ & 0.463 \\
\hline \multicolumn{5}{|l|}{ Living residency } \\
\hline \multicolumn{5}{|l|}{ Rural (ref) } \\
\hline Urban & $0.67(0.36-1.25)$ & 0.204 & $0.90(0.43-1.91)$ & 0.790 \\
\hline \multicolumn{5}{|l|}{ Birth outcomes } \\
\hline \multicolumn{5}{|l|}{ Birth length } \\
\hline \multicolumn{5}{|l|}{$<48 \mathrm{~cm}$ (ref) } \\
\hline$\geq 48 \mathrm{~cm}$ & $0.47(0.24-0.90)$ & $0.022^{*}$ & $0.36(0.16-0.83)$ & $0.016^{*}$ \\
\hline \multicolumn{5}{|l|}{ Birth weight } \\
\hline \multicolumn{5}{|l|}{$<2500 \mathrm{~g}$ (ref) } \\
\hline$\geq 2500 \mathrm{~g}$ & $0.67(0.20-2.20)$ & 0.505 & & \\
\hline \multicolumn{5}{|l|}{ Gestational age } \\
\hline \multicolumn{5}{|l|}{$<37$ wk (ref) } \\
\hline$\geq 37 \mathrm{wk}$ & $0.86(0.27-2.68)$ & 0.791 & & \\
\hline \multicolumn{5}{|l|}{ Feeding practices } \\
\hline Exclusive breastfe & & & & \\
\hline Yes (ref) & & & & \\
\hline No & & & $1.48(0.66-3.32)$ & 0.338 \\
\hline $\begin{array}{l}\text { Introduction of cor } \\
\text { food }\end{array}$ & & & & \\
\hline$<6$ months (ref) & & & & \\
\hline$\geq 6$ months & $0.75(0.35-1.58)$ & 0.449 & & \\
\hline
\end{tabular}


Table 3. Bivariate and multivariate results of factors associated with stunting [Cont'd]

\begin{tabular}{|c|c|c|c|c|}
\hline Variables & COR & $p$ & $A O R$ & $p$ \\
\hline \multicolumn{5}{|c|}{$\begin{array}{l}\text { Energy intake from } \\
\text { complementary food } \\
\quad>80 \% \text { RDA (ref) }\end{array}$} \\
\hline$<80 \% \mathrm{RDA}$ & $1.62(0.78-3.36)$ & 0.198 & $1.03(0.33-3.21)$ & 0.956 \\
\hline \multicolumn{5}{|c|}{$\begin{array}{l}\text { Protein intake from } \\
\text { complementary food } \\
\geq 80 \% \text { RDA (ref) }\end{array}$} \\
\hline$<80 \% \mathrm{RDA}$ & $1.31(0.57-3.01)$ & 0.527 & $1.58(0.40-6.26)$ & 0.510 \\
\hline \multicolumn{5}{|c|}{$\begin{array}{l}\text { Calcium intake from } \\
\text { complementary food }\end{array}$} \\
\hline$<80 \% \mathrm{RDA}$ & $1.30(0.69-2.47)$ & 0.417 & $0.96(0.33-2.75)$ & 0.933 \\
\hline \multicolumn{5}{|c|}{$\begin{array}{l}\text { Iron intake from complementary } \\
\text { food }\end{array}$} \\
\hline \multicolumn{5}{|l|}{$\geq 80 \%$ RDA (ref) } \\
\hline$<80 \% \mathrm{RDA}$ & $0.86(0.46-1.61)$ & 0.631 & 0.97 (0.31-3.04) & 0.954 \\
\hline \multicolumn{5}{|c|}{$\begin{array}{l}\text { Phosphorus intake from } \\
\text { complementary food } \\
>80 \% \text { RDA (ref) }\end{array}$} \\
\hline$<80 \% \mathrm{RDA}$ & $1.13(0.57-2.22)$ & 0.729 & $0.80(0.25-2.55)$ & 0.707 \\
\hline \multicolumn{5}{|c|}{$\begin{array}{l}\text { Vitamin D intake from } \\
\text { complementary food }\end{array}$} \\
\hline $\begin{array}{c}<80 \% \text { RDA } \\
\text { Dietary diversity }\end{array}$ & $3.03(0.92-9.98)$ & 0.068 & $5.18(1.03-26.02)$ & $0.046^{*}$ \\
\hline \multicolumn{5}{|c|}{$<4$ food groups (ref) } \\
\hline$\geq 4$ food groups & $0.55(0.15-1.95)$ & 0.354 & $0.17(0.03-0.92)$ & $0.040^{*}$ \\
\hline \multicolumn{5}{|l|}{ Infectious diseases } \\
\hline \multicolumn{5}{|l|}{ Fever } \\
\hline \multicolumn{5}{|l|}{ No (ref) } \\
\hline Yes & $1.62(0.68-3.86)$ & 0.279 & & \\
\hline \multicolumn{5}{|l|}{ Cough } \\
\hline \multicolumn{5}{|l|}{ No (ref) } \\
\hline Yes & $1.13(0.43-2.94)$ & 0.807 & & \\
\hline \multicolumn{5}{|l|}{ Flu } \\
\hline \multicolumn{5}{|l|}{ No (ref) } \\
\hline Yes & $1.24(0.50-3.05)$ & 0.646 & & \\
\hline \multicolumn{5}{|l|}{ Diarrhoea } \\
\hline No (ref) & & & & \\
\hline Yes & $0.79(0.20-3.06)$ & 0.732 & & \\
\hline
\end{tabular}

${ }^{*} p<0.005$

COR: crude odds ratio; AOR: adjusted odds ratio; RDA: recommended dietary allowance 
in the group of children who consumed $\geq 4$ food groups compared to those who had $<4$ food groups of dietary diversity.

\section{DISCUSSION}

Our results showed that low dietary diversity was associated with stunting. A significant association between dietary diversity and childhood stunting was documented previously in an earlier studies (Paramashanti et al., 2017). Dietary diversity is a good indicator of micronutrient density in the diet, thus reflecting diet quality (\%) (Moursi et al., 2008). Nonetheless, apart from micronutrient sufficiency, dietary diversity has a positive effect on stunting as we found vitamin $\mathrm{D}$ was the only micronutrient linked with stunting. Dietary diversity may also capture energy and macronutrient intakes as one eats different sources of foods (Muslimatun \& Wiradnyani, 2016), but there was no relationship between energy and protein intakes with stunting in our study. It becomes important to consider other factors such as gut microbiome, which could mediate the pathway between a diversified diet and a child's linear growth. Some studies suggested that greater dietary diversity promotes a more diverse gut microbial community in the human body, thus affecting linear growth (Reese \& Dunn, 2018; Robertson et al., 2019).

In this study, we found that children whose intakes of vitamin D were below the recommended guideline were more likely to become stunted. This result confirmed a previous study conducted in low- and middle-income setting (Mokhtar et al., 2018). Meanwhile, some trials have reported different results (Ganmaa et al., 2017; Hyppönen et al., 2011). The prevalence of inadequate vitamin D intake ( $<80 \%$ Indonesian RDA or $<480 \mathrm{IU}$ ) were present in both case (94.94\%) and control groups (86.08\%) of our study. Throughout childhood, the risk of deficiency among both males and females may increase significantly due to dietary intake insufficiency and poor access to sunshine (Koo \& Walyat, 2013; Viljakainen \& Hyppönen, 2013). Vitamin D is an essential micronutrient for bone mineralisation, growth, and development (Koo \& Walyat, 2013). However, hypovitaminosis D, if not addressed while growing, could give negative impacts on growth and development (Viljakainen \& Hyppönen, 2013).

Human growth is a multifaceted process beginning in the foetal period and ending in adolescence. In children, linear growth is regulated by growth hormone $(\mathrm{GH})$ and insulin-like growth factor (IGF-1) (Esposito et al., 2019). Vitamin D may have a direct influence on GH/IGF-1 axis but the interplay between both remains incompletely understood (Ciresi \& Giordano, 2017). Indirectly, vitamin $\mathrm{D}$ plays some roles in calcium metabolism, which is also important in stature growth. However, there was no significant relationship between calcium intake and stunting in this study.

Birth length and stunting were linked in this research. Short-statured neonates may grow as stunted children if they are not provided with adequate breastfeeding and complementary foods, proper stimulation and nurturing, and prevention practices of infectious illnesses (Prendergast \& Humphrey, 2014). Consequently, infants born with a short length may not have the opportunity for catch-up growth during the window of opportunity period. As many previous studies have concluded (Aryastami et al., 2017), this could be explained by the intergenerational process of linear growth. A study in Myanmar showed that not only low birth weight, but also short birth length, increased the risk of stunting (Khaing et al., 2019). The Bogor Longitudinal Study 
on Child Growth and Development which was conducted in Bogor City, Indonesia to study the dominant risks of stunting among 650 children aged 0-23 months found that birth weight, length at birth and maternal short stature were the most dominant risks of developing stunting (Utami et al., 2018). However, in this current study, we found no relationship between birth weight and stunting status. Having a short birth length might indicate poor nutritional status during pregnancy, where mothers had inadequate energy and micronutrient requirements (Paramashanti et al., 2017; Utami et al., 2018). This can be worsened if they had low-quality antenatal care during pregnancy (Eka et al., 2018). Prenatal nutrition is an important period as stunting often begins in utero. Therefore, optimum nutrition is strongly suggested even before conception as it has a vital role to promote optimum growth in utero that will be represented in the outcomes of a newborn's birth size and should be continued for at least the first two years of post-natal life (Aryastami et al., 2017; Titaley et al., 2019).

Older children (18-23 months) were more likely to become stunted compared with younger children. A study in Afghanistan showed a higher chance of being stunted among children aged 25-59 months compared with younger age groups (Akseer et al., 2018). This indicated that height-for-age $\mathrm{Z}$-score may become lower as the child gets older, confirming that stunting is a chronic undernutrition problem. Growth faltering may occur as soon as an infant is born, but it mostly takes place between 3 and 18-24 months (Victora et al., 2010). While $20 \%$ of stunting rates that occur at birth indicate prenatal malnutrition, the prevalence increases to $58 \%$ between 18 to 23 months (de Onis \& Branca, 2016), and this later stunted growth could be explained by poor breastfeeding and complementary feeding practices. In our study, besides dietary diversity and intake of vitamin $\mathrm{D}$ that may affect stunted growth, there could be a possible explanation of early weaning practices among older children. In India, weaning children after six months of age increased the risk of stunted growth (Padmadas, Hutter \& Willekens, 2002). This finding highlighted the importance of the continuation of breastfeeding up to 2 years old as the primary nutritional source alongside complementary feeding, especially when complementary foods do not meet the nutritional values and safety criteria. Nevertheless, we did not collect data on breastfeeding continuation in this study.

There was no significant difference in exclusive breastfeeding between stunted and non-stunted children. This could be due to the influence of complementary feeding quality (Rusmil et al., 2019). Based on Black et al. (2008) in the Lancet series, the risk of becoming stunted still exists even among exclusively breastfed children if they do not receive an adequate complementary feeding both in quality and in quantity, whereas among 6-8 months and 9-10 months old infants, breastmilk can only fulfil $70 \%$ and $50 \%$, respectively, of energy requirement, which should be complemented by foods. For this issue, if an infant fails to meet the energy requirement provided from complementary foods, she/he may have a state of growth faltering (PAHO/ WHO, 2004).

Household economic status was not related to stunting. Low economic status is generally linked with consuming cheaper foods and low diversity diet. However, high economic status does not always guarantee a good nutritional status (Ibrahim \& Faramita, 2015). Results from the Indonesia Basic Health Research showed that 20\% 
of young children from middle- and higher economic status were stunted. The underlying factors included low quality of dietary intake, which was higher in carbohydrate, sugar, and fat intakes. These inappropriate practices of complementary feeding may start since the beginning of the introduction to complementary foods (NIHRD, 2019).

Living residence of young children was not associated with stunting. This may be caused by the homogeneity of geographical areas in our study locations, so there was not much difference between urban and rural areas. Busy schedules that are much more associated with urban parents is arguably proposed as a factor that could predispose children to malnutrition (Adenuga et al., 2017). On the other hand, limited access to health facilities such as primary health centres or hospitals and nutrition knowledge in rural areas may contribute to stunting problems. Although this cannot be fully explained from the data collected in this case-control study, it does suggest that the risks of stunting vary widely across communities. This needs further investigation, particularly along urbanrural divides (Samuel, 2013).

Despite the insignificant results from macronutrient and micronutrient intakes, except for vitamin D, we could not neglect the importance of these nutrients on growth. However, our data might have been influenced by recall bias from the food frequency questionnaire which we used. Both feeding practices and history of infections were self-reported by mothers, thus adding to the possibility of recall bias in this study. Moreover, we did not take into account the duration and frequency of infections, which might have limited our understanding of the association between infectious diseases and childhood stunting in this study. However, this study succeeded to cover variables at the individual level including dietary intakes, household level, and community level, which have been adjusted during the analysis, in order to provide the best data possible in relation to stunting.

\section{CONCLUSION}

In conclusion, minimum dietary diversity, vitamin $\mathrm{D}$ intake from complementary foods, and birth length were associated with stunting among young children in Bantul District, Yogyakarta Special Region, Indonesia. Therefore, promoting adequate and varied dietary intakes with a focus in the first thousand days of life since preconception is highly recommended to improve child nutritional status.

\section{Acknowledgements}

The authors would like to acknowledge the Ministry of Research, Technology, and Higher Education of Indonesia Republic for the research funding under the scheme of dosen pemula 2018/2019 (Grant No: $111 /$ A/SP2H/DRPM/2019), with the project name of Indonesian early career lecturers research grant. Special thanks to all selected Posyandu and midwives in the public health centres of Bantul District.

\section{Authors' contributions}

EN, principal investigator, conceptualised and designed the study, prepared the draft of the manuscript and reviewed the manuscript; BAP, led the data collection in Bantul, data analysis and interpretation, and reviewed the manuscript; $\mathrm{DA}$, led the data collection in Bantul and reviewed the manuscript; ASA, assisted in drafting of the manuscript and reviewed the manuscript.

\section{Conflict of interest}

The author(s) declare no potential conflicts of interest with respect to the research, authorship, and/or publication of this article.

\section{References}

Adenuga WU, Obembe TA, Odebunmi KO \& Asuzu MC (2017). Prevalence and determinants of stunting among primary school children in rural and urban communities in obafemi owode local government area, Southwestern Nigeria. Ann Ib Postgrad Med 15(1):7-15. 
Akseer N, Bhatti Z, Mashal T, Soofi S, Moineddin R, Black RE \& Bhutta ZA (2018). Geospatial inequalities and determinants of nutritional status among women and children in Afghanistan: an observational study. The Lancet Glob Health 6(4):e447-e459.

Aryastami NK, Shankar A, Kusumawardani N, Besral B, Jahari AB \& Achadi E (2017). Low birth weight was the most dominant predictor associated with stunting among children aged 12-23 months in Indonesia. BMC Nutrition 3(1): 16 .

Beal T, Tumilowicz A, Sutrisna A, Izwardy D \& Neufeld LM (2018). A review of child stunting determinants in Indonesia. Maternal Child Nutr 14(4):e12617.

Black RE, Allen LH, Bhutta ZA, Caulfield LE, de Onis M, Ezzati M, Mathers C \& Rivera J (2008). Maternal and child undernutrition: global and regional exposures and health consequences. The Lancet 371(9608):243-260.

Chairunnisa E, Kusumastuti AC \& Panunggal B (2018). Asupan vitamin D, kalsium dan fosfor pada anak stunting dan tidak stunting usia 12-24 bulan di Kota Semarang. J Nutr Coll $7(1): 39-44$.

Ciresi A \& Giordano C (2017). Vitamin D across growth hormone $(\mathrm{GH})$ disorders: From $\mathrm{GH}$ deficiency to GH excess. Growth Horm IGF Res 33:35-42.

de Onis M \& Branca F (2016). Childhood stunting: a global perspective. Maternal Child Nutr 12(Suppl 1):12-26.

Eka R, Erlina ADP, Ina P, Erna R, Resita N, Sri L, Putri Sari W \& Muhammad Ridho N (2018). Determinants of stunting children (0-59 months) in some countries in Southeast Asia. Jurnal Kesehatan Komunitas 4(3):88-94.

Esposito S, Leonardi A, Lanciotti L, Cofini M, Muzi G \& Penta L (2019). Vitamin D and growth hormone in children: a review of the current scientific knowledge. J Transl Med 17(1):87.

Ganmaa D, Stuart JJ, Sumberzul N, Ninjin B, Giovannucci E, Kleinman K, Holick MF, Willett WC, Frazier LA \& Rich-Edwards JW (2017). Vitamin D supplementation and growth in urban Mongol school children: Results from two randomized clinical trials. PLOS ONE 12(5):e0175237-e0175237.

Hyppönen E, Fararouei M, Sovio U, Hartikainen AL, Pouta A, Robertson C, Whittaker JC \& Jarvelin MR (2011). High-dose vitamin D supplements are not associated with linear growth in a large Finnish cohort. J Nutri 141(5):843-848.
Ibrahim IA \& Faramita R (2015). Hubungan faktor sosial ekonomi keluarga dengan kejadian stunting anak usia 24-59 bulan di Wilayah Kerja Puskesmas Barombong Kota Makassar tahun 2014. Al-Sihah: Public Health Science Journal 7(1):63-75.

Khaing HT, Nomura S, Yoneoka D, Ueda P \& Shibuya K (2019). Risk factors and regional variations of malnutrition among children under 5 in Myanmar: cross-sectional analyses at national and subnational levels. BMJ Open 9(9): e030894.

Koo W \& Walyat N (2013). Vitamin D and skeletal growth and development. Curr Osteoporos Rep 11(3):188-193.

MOH Indonesia (2013). Angka kecukupan gizi yang dianjurkan bagi bangsa Indonesia. Peraturan Menteri Kesehatan Republik Indonesia Nomor 75 Tahun 2013, Ministry of Health Indonesia.

$\mathrm{MOH}$ Indonesia (2017). In: Health Profile of Yogyakarta Special Region 2017. From https: / / www.kemkes.go.id/resources / download/ profil/PROFIL_KES_PROVINSI_2017/14_ DIY_2017.pdf. [Retrieved September 25 2019].

Mokhtar RR, Holick MF, Sempértegui F, Griffiths JK, Estrella B, Moore LL, Fox MP \& Hamer DH (2018). Vitamin D status is associated with underweight and stunting in children aged 6-36 months residing in the Ecuadorian Andes. Public Health Nutr 21(11):1974-1985.

Moursi MM, Arimond M, Dewey KG, Trèche S, Ruel MT \& Delpeuch F (2008). Dietary diversity is a good predictor of the micronutrient density of the diet of 6- to 23-month-old children in Madagascar. J Nutr 138(12):2448-2453.

Muslimatun S \& Wiradnyani LAA (2016). Dietary diversity, animal source food consumption and linear growth among children aged 1-5 years in Bandung, Indonesia: a longitudinal observational study. Br J Nutr 1-9.

NIHRD (2013). In: Laporan Nasional Riskesdas 2013: National Institute of Health and Research Development (Balitbangkes). [Retrieved January 16 2019].

NIHRD (2019). In: Hasil Utama Riskesdas 2018: National Institute of Health and Research Development (Balitbangkes). From http: / /www.kesmas.kemkes.go.id/assets / upload/dir_519d41d8cd98f00/files / Hasilriskesdas-2018_1274.pdf?opwvc $=1$. [Retrieved September 27 2019]. 
Padmadas SS, Hutter I \& Willekens F (2002). Weaning initiation patterns and subsequent linear growth progression among children aged 2-4 years in India. Int $J$ Epidemiol 31(4):855863.

PAHO/WHO 2004. Guiding principles for complementary feeding of the breastfed child. Geneva, Switzerland: PAN American Health Organization/World Health Organization.

Paramashanti BA, Hadi H \& Gunawan IMA (2015). Pemberian ASI eksklusif tidak berhubungan dengan stunting pada anak usia 6-23 bulan di Indonesia. J Gizi Dietetik Indones (IJND) 3(3):162-174.

Paramashanti BA, Paratmanitya Y \& Marsiswati (2017). Individual dietary diversity is strongly associated with stunting in infants and young children. J Gizi Klinik Indones 14(1):19-26.

Prendergast AJ \& Humphrey JH (2014). The stunting syndrome in developing countries. Paediatr Int Child H 34(4):250-265.

Ramadhani NA, Bahar B \& Dachlan DM (2019). Hubungan asupan zat gizi makro dan zat gizi mikro dengan stunting pada anak usia 2459 bulan di wilayah kerja Puskesmas Kabere Kecamatan Cendana Kabupaten Enrekang. JGMI: The Journal of Indonesian Community Nutrition 8(2):90-97.

Reese AT \& Dunn RR (2018). Drivers of microbiome biodiversity: A review of general rules, feces, and ignorance. mBio 9(4):e01294-18.

Robertson RC, Manges AR, Finlay BB \& Prendergast AJ (2019). The human microbiome and child growth - first 1000 days and beyond. Trends Microbiol 27(2):131-147.

Rusmil V, Prahastuti T, Dimas E, Luftimas D \& Hafsah T (2019). Exclusive and Non-exclusive breastfeeding among stunted and normal 6-9 month-old-children in Jatinangor Subdistrict, Indonesia. Althea Med J 6:35-41.

Samuel FO (2013). Pattern of stunting among under-five children in selected urban and rural communities in Oyo State, Nigeria. ICAN: Infant, Child Adolesc Nutr 5(6):393-398.
Sebayang SK, Dibley MJ, Astutik E, Efendi F, Kelly PJ \& Li M (2019). Determinants of ageappropriate breastfeeding, dietary diversity, and consumption of animal source foods among Indonesian children. Maternal Child Nutri 16(1):e12889.

Titaley CR, Ariawan I, Hapsari D, Muasyaroh A \& Dibley MJ (2019). Determinants of the stunting of children under two years old in Indonesia: A multilevel analysis of the 2013 Indonesia Basic Health Survey. Nutrients 11(5):1106.

Utami N, Rachmalina R, Irawati A, Sari K, Rosha BC, Amaliah N \& Besral (2018). Short birth length, low birth weight and maternal short stature are dominant risks of stunting among children aged 0-23 months: Evidence from Bogor longitudinal study on child growth and development, Indonesia. Mal J Nutr 24:11-23.

Victora CG, De Onis M, Hallal PC, Blössner M \& Shrimpton R (2010). Worldwide timing of growth faltering: Revisiting implications for interventions. Pediatrics 125(3):e473-e480.

Viljakainen H \& Hyppönen E (2013). Vitamin $\mathrm{D}$ and growth. In Watson R. (ed.) Handbook of Vitamin D in Human Health: Prevention, Treatment and Toxicity. Wageningen Academic Publishers, Wageningen.

WHO (2006). In: WHO Child Growth Standards: length/height-for-age, weight-for-age, weightfor-length, weight-for-height and body mass index-for-age: methods and development. World Health Organization. From https://apps.who. int/iris/handle/10665/43413. [Retrieved September 3 2019].

WHO (2008). In: Indicators for assessing infant and young child feeding practices: part 1 . definitions: World Health Organization.

Yu EA, Huey SL, Peña-Rosas JP \& Mehta S (2017). The effects of oral vitamin D supplementation on linear growth and non-communicable diseases among infants and children younger than five years of age. Cochrane Database Syst Rev 2017(11): CD012875. 